\title{
Samuel J. Fomon - Champion de la croissance
}

\author{
Ekhard E. Ziegler Ferdinand Haschke
}

Samuel J. Fomon s'est intéressé à la croissance pendant toute sa carrière, ce qui l'a distingué de la plupart des autres chercheurs dans le domaine de la nutrition. Il est hors de question d'amoindrir ses nombreuses autres contributions, mais l'élucidation de la composition corporelle et de ses changements au cours de l'enfance et l'introduction de la croissance comme indicateur de l'état nutritionnel se distinguent comme ses contributions les plus originales et sans doute les plus importantes. Même s'il n'avait accompli que cela, sa place au panthéon des sciences de la nutrition n'en serait pas moins assurée. Ses réalisations ont été bien entendu beaucoup plus nombreuses.

Samuel J. Fomon est décédé le 18 décembre 2007 après avoir longuement et vaillamment lutté contre un myélome multiple. Il est né le 9 mars 1923 à Chicago. Après le décès prématuré de sa mère, il passe son enfance chez des parents dans le Wisconsin, où il fréquente également une école secondaire. En 1944, il est diplômé cum laude de l'Université d'Harvard et, en 1947, reçoit son diplôme de médecin de l'Université de Pennsylvanie. Après une formation à titre d'interne en pédiatrie au Children's Hospital de Philadelphie, il termine des études en physiologie rénale au Children's Hospital de Cincinnati dans le cadre d'une bourse de recherche. Il sert comme capitaine dans l'armée américaine pendant deux ans avant de rejoindre le service de pédiatrie de l'université de l'Iowa en 1954. Bien que devenu professeur émérite en 1993, il a continué à mener très activement des recherches pendant une décennie supplémentaire. Il a occupé de nombreux postes importants et notamment ceux de Président du Comité de nutrition de l'American Academy of Pediatrics (1960-63), de conseiller, de membre du comité de rédaction et, par la suite, de Président (1981-82) de l'American Society of Clinical Nutrition, et de membre du comité de rédaction, de conseiller et finalement de Président de l'American Institute of Nutrition. Parmi les nombreuses distinctions qu'il a reçues, le Bristol-Meyers Squibb/Mead Johnson Award (1992) est probablement la plus prestigieuse. Il a attiré des dizaines de stagiaires et de post-doctorants venus du

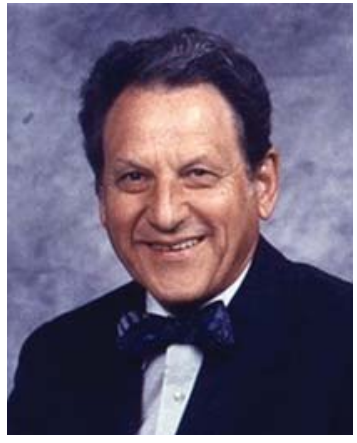

monde entier et a formé de nombreux étudiants en doctorat. La plupart repartent différents, certains plus, d'autres moins, mais tous munis d'une boussole les orientant vers une nutrition infantile sensée.

Peu de temps après son arrivée dans l'Iowa, le Dr Fomon est invité à prendre la tête de l'unité métabolique en activité depuis les années 1920. Commence alors un voyage de cinq décennies à travers la nutrition infantile. Parvenu à la fin de ce parcours, le Dr Fomon avait totalement transformé le domaine. Il passe ses premières années à l'Université de l'Iowa à mener des études sur l'équilibre métabolique dans l'optique de déterminer les besoins en nutriments des nourrissons. Réalisant que les études d'équilibre sont mal adaptées à cette fin et que la croissance est la force motrice sous-tendant les besoins nutritionnels élevés du jeune enfant, il commence à consacrer une grande partie de son énergie à l'étude de la croissance et fait des contributions fondatrices dans deux domaines. La composition de l'organisme est le premier d'entre eux. Dans une flambée d'énergie créatrice, il construit à partir de données limitées un premier projet de ce qui allait devenir le nourrisson de référence. Le modèle est immédiatement mis à profit pour déterminer les besoins en nutriments par la méthode factorielle, une autre importante création aux conséquences considérables.

\section{KARGER}

Fax +41613061234 E-Mail karger@karger.ch www.karger.com
(C) 2008 Nestec Ltd., Vevey/S. Karger AG, Basel 0250-9644/07/0653-0095\$23.50/0 
Viennent ensuite le développement et l'application de méthodes de mesure précises et reproductibles de la croissance. Grâce à ces méthodes, il devient possible, entre autres, d'utiliser la croissance comme indicateur de l'état nutritionnel. Il est juste de dire que l'introduction de mesures normalisées de la croissance a joué un rôle important dans le développement de la nutrition infantile, dont le domaine d'investigation est passé du qualitatif au quantitatif.

Les travaux sur la composition corporelle ont débuté à partir d'un ensemble d'informations très limitées, qui comprenaient des données provenant d'analyses chimiques de mortnés et de quelques adultes, ainsi que des données portant sur l'eau corporelle totale et la composition des tissus. La première version du nourrisson de référence de sexe masculin est présentée en 1966 [1]. Elle est rapidement suivie d'une révision majeure fondée sur de nouvelles données [2]. Cette réalisation, le nourrisson de référence de 1967, a résisté à l'épreuve du temps. Elle a permis l'estimation des besoins en protéines et autres nutriments par la méthode factorielle. Tout aussi importants ont été les aperçus qu'elle a permis, révélant par exemple que jusqu'à la moitié des protéines consommées au cours des premiers mois de vie est consacrée à la croissance, ou que $40 \%$ de la prise de poids d'un nourrisson sont dus à l'accumulation de graisses, ce qui explique pourquoi les apports énergétiques sont si élevés au début de la vie. La méthode factorielle a alimenté et continue à féconder d'autres domaines tels que la nutrition des nourrissons prématurés $[3,4]$.

Des données sur le potassium corporel total et sur le contenu minéral osseux sont devenues disponibles au cours des années suivantes. Ces nouvelles informations ont permis la construction d'un garçon de référence âgé de neuf ans [5]. Elles ont également abouti à une refonte du nourrisson de référence et à une extension sans lacune jusqu'à l'âge de 10 ans (enfants de référence), cette fois pour les filles comme pour les garçons [6]. Un impor-tant concept issu de ces travaux a été que la densité de la masse corporelle non grasse des enfants était sensiblement inférieure à celle des adultes, ce qui a eu d'importantes impli- cations pour la détermination de la masse grasse par des méthodes telles que l'hydrodensitométrie et la pléthysmographie, qui reposent sur des hypothèses liées à la densité de la masse non grasse. Le Dr Fomon a continué à étudier la composition corporelle et ce n'est qu'en 2002 qu'il a résumé l'œuvre de sa vie en ce domaine [7].

Afin d'obtenir des mesures reproductibles de la croissance et d'améliorer l'interprétabilité des données la concernant, le Dr Fomon a insisté sur la nécessité de prêter une attention méticuleuse aux techniques de mesure et, surtout, à l'utilisation d'intervalles d'âge rigoureusement normalisés. Il a également insisté sur l'utilisation de données incrémentielles plutôt que du poids ou de la taille atteints à titre de meilleur moyen d'analyser et d'interpréter des données sur la croissance. Des mesures normalisées de la croissance ont commencé à être utilisées vers le milieu des années 1960 [par exemple, 8] et ont continué à l'être avec un grand succès afin de répondre à des questions importantes comme celle de l'effet de la concentration d'une formule sur la croissance des nourrissons en bonne santé [9] et à de nombreuses autres. L'amélioration des méthodes de mesure a ainsi permis l'utilisation de la croissance comme critère lors d'études nutritionnelles et a considérablement élargi l'éventail des questions auxquelles il devenait possible de répondre par de simples études bien menées sur la croissance.

En 1988, la Food and Drug Administration des États-Unis a demandé l'avis de l'American Academy of Pediatrics sur les essais cliniques de formules pour nourrissons. Le Dr Fomon a préparé alors la réponse de l'AAP. Il a recommandé, entre autres mesures, des études normalisées de la croissance comme critères ultimes de la valeur nutritionnelle des formules pour nourrissons. La FDA a adopté alors ces recommandations dans leur intégralité et suit encore à ce jour les règles édictées en 1988 par le Dr Fomon.

La croissance n'est pas le seul domaine où l'énergie créatrice du Dr Fomon a laissé une empreinte indélébile. Mais en termes d'innovation globale et d'authentique impact, son travail sur la croissance constitue son principal titre de gloire.

\section{Références}

1 Fomon SJ: Body composition of the infant. Part I: The male 'reference infant'; in Falkner F (ed): Human Development. Philadelphia, Saunders, 1966, pp 239-246.

2 Fomon SJ: Body composition of the male reference infant during the first year of life. Pediatrics 1967;40:863-870.

3 Fomon SJ, Ziegler EE, Vazquez HD: Human milk and the small premature infant. Am J Dis Child 1977;131:463-467.
4 Ziegler EE: Protein requirements of very low birth weight infants. J Pediatr Gastroenterol Nutr 2007;45:S170-S174.

5 Haschke F, Fomon SJ, Ziegler EE: Body composition of a nine-year-old reference boy. $\mathrm{Pe}$ diatr Res 1981;15:847-849.

6 Fomon SJ, Haschke F, Ziegler EE, et al: Body composition of reference children from birth to age 10 years. Am J Clin Nutr 1982;35: $1169-1175$.
7 Fomon SJ, Nelson SE: Body composition of the male and female reference infants. Annu Rev Nutr 2002;22:1-17.

8 Fomon SJ, Younoszai, MK, Thomas, LN: Influence of vitamin $\mathrm{D}$ on linear growth of normal full-term infants. J Nutr 1966;88: 345-350.

9 Fomon SJ, Filer LJ Jr, Thomas LN, Rogers RR, Proksch AM: Relationship between formula concentration and rate of growth of normal infants. J Nutr 1969;98:241-254. 